\title{
Non-conventional optimization techniques in optimizing non-traditional machining processes:
} A review

\author{
Arindam Debroy and Shankar Chakraborty ${ }^{*}$
}

Department of Production Engineering, Jadavpur University, Kolkata - 700 032, INDIA

\section{H R O N I C L E}

Article history:

Received April 23, 2012

Received in revised format

22 October 2012

Accepted 26 October 2012

Available online

October 312012

Keywords:

Non-traditional machining

process

Non-conventional optimization

technique

Machining parameter

Response \begin{abstract}
A B S T R A C T
With the ever-increasing demands for high surface finish and complex shape geometries, conventional metal removal methods are now being replaced by non-traditional machining (NTM) processes. These NTM processes use energy in its direct form to remove materials in the form of atoms or molecules to obtain the required accuracy and burr-free machined surface. In order to exploit the optimal capabilities of the NTM processes, it is often required to determine the best possible combinations of their controllable parameters. Different nonconventional optimization techniques have been used for dealing with these process optimization problems because of their inherent advantages and capabilities for arriving at the almost global optimal solutions. This paper reviews the applications of different nonconventional optimization techniques for parametric optimization of NTM processes. It is observed that electrical discharge machining processes have been optimized most number of times, followed by wire electrical discharge machining processes. In most of the cases, the past researchers have preferred to maximize material removal rate. Genetic algorithm has been found to be the most popular non-conventional optimization technique.
\end{abstract}

C) 2013 Growing Science Ltd. All rights reserved.

\section{Introduction}

The advent of newer materials and need for better surface finish along with high dimensional accuracy have paved the way for increased applications of different non-traditional machining (NTM) processes in manufacturing industries. Each of these NTM processes has several controllable parameters, which primarily influence the process performance measures (responses). Choosing the appropriate process parameters and their corresponding levels to achieve the target responses can only be possible after having the knowledge about how these parameters influence the process performance. Thus, selection of the optimal parametric combination for any NTM process is an important task for having increased production rate with reduced machining time. Determination of the optimal NTM process parameters becomes also difficult due to the presence of complex

*Corresponding author. Tel: 091-033-2414-6153

E-mail addresses: s_chakraborty00@yahoo.co.in (S. Chakraborty) 
stochastic physico-chemical and thermal phenomena in the machining zone. Suitable selection of NTM process parameters depends on the operator's technological knowledge and experience because of their numerous and diverse range. The values of different NTM process parameters provided by the manufacturers often cannot meet the operator's requirements. For a given workpiece material and shape feature combination, the desired parametric setting cannot be obtained from the manufacturers' handbook. Sometimes, the chosen process parameters are conservative and far from the optimal, constraining the full utilization of the NTM process capabilities. Thus, it is undoubtedly a challenge to ensure that the selected process parameters result in optimal or near optimal machining performance of NTM processes which encourages the application of various optimization tools in this domain. Although several optimization techniques, like Taguchi method, desirability function approach, grey relational analysis, fuzzy logic etc. have been successfully applied to optimize NTM processes, it is observed that the applications of different non-conventional optimization techniques have been quite appealing and promising to attain the best performance of NTM processes.

Most of the conventional optimization techniques are based on gradient search methods and have the possibility of getting trapped at the local optimum depending upon the degree of non-linearity in the objective function and initial guess about the starting point. Hence, these conventional optimization techniques do not ensure global optimum and thus have limited applications. In the recent past, various non-conventional search and optimization methods based on natural phenomena (evolutionary computation), like genetic algorithm (GA), simulated annealing (SA), differential evolution (DE), ant colony optimization (ACO), artificial bee colony (ABC), harmony search (HS), sheep flock algorithm (SFA), particle swarm optimization (PSO), biogeography-based optimization (BBO) etc. have been developed to overcome this problem. Moreover, when an optimization problem contains multiple global solutions, the users are not only interested in finding just one global optimal solution, but as many as possible equally good solutions for later use. However, if the conventional methods are used to find out multiple optimal solutions, they need to be applied a given number of times, each time starting from a different initial guess point and hoping to achieve a different optimal solution.

Harnessing the non-conventional optimization techniques with a computer provides a means for addressing complex engineering problems with chaotic disturbances, randomness and complex nonlinear dynamics that the conventional algorithms have been unable to conquer. The task of a nonconventional optimization technique is thus to avoid the bad solutions in the search space, take clues from the good solutions and eventually reach close to the best solution. Non-conventional algorithmbased optimizers are global optimization methods and scale well to higher dimensional problems with multiple solutions. These algorithms are robust and can easily be adjusted according to the problem at hand. Almost any aspect of the algorithm can be changed and customized. For example, in the case of GA, it is better than the conventional optimization methods in the following aspects,

a) It does not use gradient information.

b) It works with a set of solutions instead of one solution in each iteration.

c) It is a stochastic search process.

d) As it is a population-based optimization approach, parallel processing can be possible for arriving at the multiple feasible solutions.

Yusup et al. (2012) made a review on the application of various evolutionary optimization techniques to optimize different controllable parameters of both traditional and modern machining processes. Applications of five popularly used optimization algorithms, e.g. GA, SA, PSO, ACO and ABC algorithms were considered. It was observed from the review that GA was the widely applied evolutionary algorithm and multi-pass turning was the machining operation, considered mostly by the past researchers for application of various evolutionary algorithms. On the other hand, surface roughness (SR) was optimized most number of times. Chandrasekaran et al. (2010) reviewed the 
applications of some soft computing tools, like artificial neural network (ANN), fuzzy set, GA, SA, $\mathrm{ACO}$ and PSO techniques in the area of four machining processes, i.e. turning, milling, drilling and grinding.

In this paper, an extensive review is made on the applications of different non-conventional optimization techniques for parametric optimization of various NTM processes. It is observed that GA, SA, ABC, HS, SFA, ACO, PSO and BBO techniques have been commonly applied for optimizing the machining performance of NTM processes.

\section{Non-traditional machining processes}

In order to meet the present day requirements of manufacturing industries, several NTM processes have already been come into market having diverse machining characteristics and potentialities. Each of these NTM processes has its own merits and demerits. In this paper, the following 12 NTM processes are identified where different non-conventional optimization techniques have been successfully applied for determining the optimal parametric combinations.

\subsection{Electrochemical machining process}

Electrochemical machining (ECM) is one of the NTM processes used to machine extremely hard materials that are difficult to machine using conventional methods. The ECM process is based on the principle of material removal by electrochemical dissolution of an anode. In this process, a direct current with high amperage and low voltage is passed between the workpiece (anode) and the tool (cathode). At the anodic workpiece surface, metal is dissolved in the electrolyte as metallic ions by the electrochemical reactions, and thus the tool shape is copied on the workpiece. ECM process is widely used for a variety of engineering applications due to its various hole drilling, 3D contour making and complicated shape-producing capabilities in electrically conducting but hard-to-machine materials, such as super alloys, titanium alloys, alloy steel, tool steel, stainless steel etc. The main advantages of ECM process include no tool wear, no thermal damage to the workpiece material, good surface finish and dimensional tolerance, and burr-free and stress-free machined surface.

Rao et al. (2008) considered tool feed rate, electrolyte flow velocity and applied voltage as the most important machining parameters, and dimensional accuracy, tool life, material removal rate (MRR) and machining cost as the performance measures of an ECM process. PSO algorithm was applied to determine the optimal combination of ECM process parameters subject to constraints of temperature, choking and passivity. Both the single- and multi-objective optimization problems were modeled, and solved using PSO algorithm. Datta and Das (2010) considered current, voltage, flow rate and gap as the major control factors for an ECM process, and applied a multi-objective GA for maximizing MRR and minimizing SR. Senthilkumar et al. (2010) identified electrolyte concentration, electrolyte flow rate, applied voltage and tool feed rate as the most influencing parameters of an ECM process, and simultaneously optimized MRR and SR using non-dominated sorting genetic algorithm-II (NSGA-II).

Samanta and Chakraborty (2011) optimized an ECM and a micro-ECM process using ABC algorithm. For the ECM process, electrolyte concentration, electrolyte flow rate, applied voltage and inter-electrode gap were the machining parameters, whereas, MRR and overcut (OC) were the responses. In micro-ECM process, the optimal parametric settings of pulse on/off ratio, machining voltage, electrolyte concentration, voltage frequency and tool vibration frequency were determined while optimizing MRR and radial overcut (ROC). The ABC algorithm was employed for solving both single- and multi-objective optimization problems. Senthilkumar et al. (2011) developed a multiple regression model to represent relationship between the machining parameters (electrolyte 
concentration, electrolyte flow rate, applied voltage and tool feed rate) and responses (MRR and SR) of an ECM process. A multi-objective optimization method based on NSGA-II technique was used to optimize the ECM process. Mukherjee and Chakraborty (2012) applied BBO algorithm to determine the optimal machining parameters of an ECM process and a wire electrochemical turning (WECT) process. For the ECM process, electrolyte concentration, electrolyte flow rate, applied voltage and inter-electrode gap were the control parameters, and MRR and OC were considered as the responses. For WECT process, wire diameter, wire feed rate, applied voltage, workpiece rotational speed and overlap distance were the machining parameters, and MRR, SR and roundness error (RE) were the process performance measures. It was observed that the optimization performance of BBO algorithm had been better than that of GA and ABC algorithm. Jain and Jain (2012) optimized tool feed rate, electrolyte flow velocity and applied voltage of an ECM process with an objective to minimize geometrical inaccuracy subjected to temperature, choking and passivity constraints using real-coded GA.

\subsection{Electrical discharge machining process}

Electrical discharge machining (EDM) is a widely used NTM process where thermal energy of the spark is used to machine electrically conductive as well as non-conductive materials regardless of their hardness. It is a machining process based on the principle of controlled application of highfrequency electric discharge onto a workpiece that conducts electricity thus detaching small particles from the workpiece by melting and evaporating them. It offers a way to process materials of very complex geometry with very fine and high precision, and has the distinct advantage in the manufacture of mould, die, automotive, aerospace and surgical components. Basically, this process involves the phenomena of spark initiation, dielectric breakdown and thermo-mechanical erosion of metals. It has been observed that the presence of suspended particles in the dielectric fluid of EDM process would significantly increase the surface finish and machining efficiency of the process. Micro-electrical discharge machining ( $\mu$-EDM), a modification of EDM process, has the capacity to machine complex three-dimensional shapes on any electrically conductive material regardless of its hardness, strength and toughness.

Fenggou and Dayong (2004) applied ANN technique to determine and optimize the machining parameters (current peak value and pulse width-on) in an EDM sinking process. GA was employed to optimize and train the developed ANN to enhance the training speed. Processing depth was the considered response. Su et al. (2004) applied GA adapted to ANN to determine the optimal EDM process parameters, like pulse-on time, pulse-off time, high-voltage discharge current, low-voltage discharge current, gap size, servo-feed, jumping time and working time. MRR, SR and tool wear rate (TWR) were the responses. The GA-based NN model could be able to determine the optimal process parameters from rough cutting stage to finish cutting stage. Mandal et al. (2007) applied NSGA-II technique to optimize multiple responses (MRR and absolute TWR) of an EDM process. Machining current, pulse-on time and pulse-off time were selected as the control parameters.

Salman and Kayacan (2008) took into consideration current, pulse-on time, pulse-off time and arc voltage as different EDM process parameters, and modeled SR by using genetic expression programming (GEP) method. The optimal combination of EDM process parameters was obtained for minimum SR value. Gao et al. (2008) integrated ANN and GA to develop a parametric optimization model for an EDM process. The ANN model was used to represent the relationship between MRR and three input parameters, e.g. current, pulse-on time and pulse-off time, and GA was finally employed to optimize those parameters. Kolahan and Bironro (2008) considered grain size of aluminum powder, concentration of the powder, discharge current and pulse-on time as the important machining parameters of a powder-mixed EDM process. The process responses were MRR and electrode wear rate (EWR). GA was used to search out the optimal process parameters within the set limiting values. Kanagarajan et al. (2008) investigated the effects of four machining parameters of an 
EDM process, such as pulse current, pulse-on time, electrode rotation and flushing pressure on MRR and SR. NSGA-II method was used for parametric optimization of the EDM process.

Rao et al. (2009) optimized SR of a die sinking EDM process by considering the simultaneous effects of two machining parameters, i.e. peak current and voltage while incorporating a multi-perceptron neural network. GA was applied to optimize the weighting factors of the developed $\mathrm{NN}$ and it was found that GA could reduce the error down to less than $2 \%$ from more than $5 \%$. Joshi and Yang et al. (2009) applied SA algorithm for selecting the best combination of machining parameters in an EDM process. MRR was maximized and SR was minimized. The selected process parameters were discharge current, source voltage, pulse-on time and pulse-off time. Somashekhar et al. (2010) applied a feed forward NN with back propagation (BP) algorithm for analyzing the material removal of micro-EDM process to establish a parameter optimization model. GA was employed to determine the optimal process parameters (gap voltage, capacitance, feed rate and speed) for any desired output value of the considered machining characteristics.

Pande (2011) developed an intelligent approach for modeling and optimization of EDM process by integrating finite element method with ANN and GA to improve prediction accuracy of the proposed model. An ANN-based process model was also proposed to establish relation between the input process conditions (current, discharge voltage, duty cycle and discharge duration) and process responses (crater size, MRR and TWR). The developed ANN model along with NSGA-II method was adopted to select the optimal parametric setting of the EDM process. Maji and Pratihar (2011) investigated the effects of peak current, pulse-on time and pulse duty factor on MRR and SR for an EDM process. The developed regression model was solved using GA to determine the optimal parametric combinations. Nejad (2011) studied the effects of discharge current, pulse-on time and pulse-off time of an EDM process on MRR and SR. An ANN with BP algorithm was adopted to model the process and it was subsequently optimized using NSGA-II algorithm.

Mukherjee and Chakraborty (2012) applied BBO algorithm for single- and multi-objective optimization of the responses of two EDM processes. For the first EDM process, peak current and pulse-on duration were the control parameters, and SR, surface crack density (SCD) and white layer thickness (WLT) were the process performance measures. In the second case, pulse-on time, peak current, average gap voltage and percent volume fraction of silicon carbide (SiC) present in the aluminum matrix were considered as the process parameters, while MRR, TWR, gap size and SR were the responses. It was further observed that the optimization performance of BBO algorithm had been better than that of GA, ACO and ABC algorithms.

Padhee et al. (2012) considered concentration of silicon powder in the dielectric fluid, pulse-on time, duty cycle and peak current as the independent variables of a powder-mixed EDM process for which the machining performance was analyzed in terms of MRR and SR. NSGA method was adopted to optimize the responses so that a set of mutually dominant solutions could be obtained over a wide range of machining parameters. Bharti et al. (2012) applied NSGA technique for optimization of a die-sinking EDM process. An ANN with BP algorithm was first used to model the EDM process. NSGA was then employed in the trained network and a set of Pareto optimal solutions was obtained. Shape factor, pulse-on time, discharge current, duty cycle, gap voltage, flushing pressure and tool electrode lift were the process parameters, and MRR and SR were the responses. Majumder (2012) optimized spark current, pulse-on duration and pulse-off duration of an EDM process for minimum EWR, while employing GA as the optimization tool.

\subsection{Electrochemical discharge machining process}

Electrochemical discharge machining (ECDM) is a hybrid NTM process taking the advantages of both ECM and EDM processes. It is a reproductive machining process in which the form of the tool 
electrode is mirrored on the workpiece surface. In this process, the thermal erosive effects of electrical discharge action follow an electrochemical reaction. This electrochemical reaction helps in the generation of positively charged ions and gas bubbles. These gas bubbles accumulate across the interface of the tool and workpiece. The electrical discharge actions take place between the tool and electrolyte across the gas bubble layers. When the applied DC power supply voltage is greater than the breakdown voltage of the insulating layer of the gas bubbles, a spark is initiated. These repetitive sparks generate minute craters on the workpiece surface, thus generating the desired shape. This hybrid process is being widely applied for machining of non-conductive materials, like ceramics.

Samanta and Chakraborty (2011) applied ABC algorithm for single- and multi-objective optimization of an ECDM process. Applied voltage, electrolyte concentration and inter-electrode gap were the considered process parameters, and MRR, ROC and thickness of heat affected zone (HAZ) were the responses. The obtained optimal values of the responses were observed to be better than those derived by the past researchers.

\subsection{Wire electrical discharge machining process}

Wire electrical discharge machining (WEDM) has become an important NTM process, widely used in aerospace, nuclear and automotive industries, for machining various hard-to-machine materials, like titanium, molybdenum, zirconium, nimonics etc. with high surface finish and intricate shapes. It is a process of removing materials from electrically conductive workpieces using thermoelectric energy. The material removal mechanism of WEDM process is based on the controlled erosion through a series of repetitive sparks between the workpiece and tool. In this process, a wire of small diameter is used as the electrode to cut a narrow width of cut in the workpiece. The wire is pulled through the workpiece from a supply spool onto a take-up mechanism. On applying a proper voltage, discharge occurs between the wire electrode and workpiece in the presence of a flood of dielectric fluid (deionized water) of high insulation resistance. The dielectric fluid is continuously injected to flush away the debris from the machining zone. Numerical control is used for precise control of the motions during the machining operation. The WEDM is an economical process and can be used to cut hard-to-machine materials with complex, precise and accurate contours in low volume and greater variety. The most important control parameters for this process are discharge current, discharge capacitance, pulse duration, pulse frequency, wire speed, wire tension, average working voltage and dielectric flushing condition, whereas, cutting velocity/MRR, workpiece surface finish/SR and kerf (cutting width) are the predominant performance measures.

Tarng et al. (1995) applied a feed forward NN and SA algorithm for attaining the optimal cutting performance of a WEDM process while considering pulse-on time, pulse-off time, peak current, noload voltage, servo reference voltage, capacitor setting and servo speed setting as the process parameters, and SR and machining speed as the responses. Kuriakose and Shunmugam (2005) considered machining voltage, spark gap, ignition pulse current, time between two pulses, pulse duration, servo-control reference voltage, maximum servo-speed variation, wire speed, wire tension and injection pressure as the important WEDM process parameters, and cutting velocity/ MRR and SR as the responses. A multi-objective optimization method based on NSGA technique was used to optimize the said WEDM process.

Mahapatra and Patnaik (2006) observed the parametric influences of discharge current, pulse duration, pulse frequency, wire speed, wire tension and dielectric flow rate on MRR and SR for a WEDM process. GA was then used to optimize the WEDM process with multiple objectives. Rao and Pawar (2009) investigated the effects of different WEDM process parameters, like pulse-on time, pulse-off time, peak current and servo feed setting on machining speed and SR. ABC algorithm was then used to determine the optimal process parameter settings for maximizing machining speed for a desired surface finish. Chen et al. (2010) proposed the integration of BPNN and SA algorithm to 
determine the optimal parametric setting of a WEDM process. Pulse-on time, pulse-off time, arc-off time, servo voltage, wire feed rate, wire tension and water pressure were the WEDM process parameters, and cutting velocity and two SR parameters ( $\mathrm{Ra}$ and $\mathrm{Rt}$ ) were the responses.

Kuruvila and Ravindra (2011) used GA for parametric optimization of WEDM process considering dimensional error (DE), SR and volumetric material removal rate (VMRR) as the performance measures. Pulse-on duration, current, pulse-off duration, bed-speed and flushing rate were the input parameters. Golshan et al. $\left(2011^{\mathrm{a}}\right)$ optimized SR and VMRR of a WEDM process employing NSGA method. The influences of electrical current, pulse-off time, open-circuit voltage and gap voltage on SR and VMRR were also studied. Kumar and Agarwal (2011) optimized MRR and SR of a WEDM process based on NSGA-II method while considering pulse peak current, pulse-on time, pulse-off time, wire feed, wire tension and flushing pressure as the important WEDM process parameters. Amini et al. (2011) used a multilayer perceptron NN for modeling of a WEDM process and investigated the effects of various process parameters on MRR and SR. GA was then employed to optimize the performance of the WEDM process.

Golshan et al. $\left(2011^{\mathrm{b}}\right)$ applied NSGA method for optimization of a WEDM process while considering electrical current, pulse-off time, open-circuit voltage and gap voltage as the major machining parameters, and SR and VMRR as the process responses. Kondayya and Gopala Krishna (2011) applied genetic programming (GP) for modeling and optimization of a WEDM process. Two WEDM process performance measures, i.e. MRR and SR were modeled based on experimental data using GP with respect to four input variables. The problem was formulated as a multi-objective optimization problem and subsequently solved using NSGA-II method. Mukherjee et al. (2012) compared the optimization performance of six non-conventional algorithms, i.e. GA, PSO, SFA, ACO, ABC and $\mathrm{BBO}$ for single- and multi-objective optimization of two WEDM processes. For the first process, peak current, duty factor, wire tension and water pressure were the process parameters, and VMRR, wear ratio (WR) and SR were the responses. While in the second WEDM process, discharge current, pulse duration, pulse frequency, wire speed, wire tension and dielectric flow rate were taken as the process parameters, whereas, MRR, SR and kerf width were considered as the responses. It was observed that $\mathrm{BBO}$ algorithm had given the best optimization performance.

Somashekhar et al. (2012) considered gap voltage, capacitance and feed rate as the control parameters, and MRR, OC and SR as the performance measures for a micro-WEDM process. The optimal parametric settings were derived using SA algorithm. Yang et al. (2012) analyzed the variations in MRR, SR and corner deviation (CD) with respect to various parameters, like pulse-on time, pulse-off time, arc-off time, servo voltage, wire feed rate, wire tension and water pressure in a WEDM process. A BPNN-integrated SA algorithm was implemented for parametric optimization of the considered process. Rao and Kalyankar (2012) adopted teaching-learning-based optimization (TLBO) algorithm to search out the optimal values of pulse-on time, pulse-off time, peak current and servo feed setting for maximizing cutting speed of a WEDM process while ensuring a constrained value of SR. A comparison with GA, SA, ABC, PSO, HS and shuffled frog leaping (SFL) algorithms had proved the superiority of TLBO algorithm over the others.

\subsection{Laser beam machining process}

Laser beam machining (LBM) is being used as a better alternative to mechanical machining processes due to its ability to machine different hard-to-machine materials and complex shape features with minimum amount of waste. In this process, there is no need of any tool because it is a non-contact machining operation. Some features, like straight/curved grooves, angled channels with straight walls and rectangular pockets with sharp corners can only be machined using this process. It operates as a sequential ablation process causing material vaporization and removal of material from the workpiece 
surface. Although this process removes much less material, it has several advantages over the other NTM processes, like no tool wear, minimum distortion of the workpiece, narrow HAZ, no residual stress generation, deep penetration and less tendency to spatter. The performance of a LBM process is usually influenced by several machining parameters, like laser power, wavelength, efficiency and emerging beam diameter.

Ciurana et al. (2009) applied a multi-objective PSO algorithm to determine the optimal values of pulse intensity, scanning speed and pulse frequency for a Nd:YAG LBM process. The objectives were to minimize SR and volume error. The relationship between the considered process parameters and responses was also modeled using an ANN.

\subsection{Ultrasonic machining process}

In ultrasonic machining (USM) process, material is removed from the workpiece surface due to the action of abrasive grains. In this process, high frequency electrical energy is converted into mechanical vibrations through a transducer, which are transmitted to the abrasive particles in the slurry through an energy-focusing device or horn/tool assembly. The tool is shaped as the approximate mirror image of the configuration of cavity desired on the workpiece surface. It can machine a large variety of materials as it is not limited by the electrical or chemical characteristics of the workpiece. This process has the advantage of machining hard and brittle materials into complex shapes with good accuracy and reasonable surface finish. Various machining parameters as involved in USM process are amplitude of tool oscillation, type of abrasive, grain size of the abrasive, feed force, volume concentration of abrasive in water slurry etc., which affect various performance measures, like MRR and SR. The USM process is characterized by low MRR and almost no surface damage to the workpiece.

Jain et al. (2007) determined the optimal values of amplitude of vibration, frequency of vibration, mean diameter of abrasive grains, volumetric concentration of abrasive particles in slurry and static feed force of an USM process using GA. MRR was maximized subject to SR constraint. Rao et al. (2010) considered amplitude of ultrasonic vibration, frequency of ultrasonic vibration, mean diameter of abrasive particles, volumetric concentration of abrasive particles and static feed force as the machining parameters of an USM process. The objective considered was maximization of MRR subjected to constraint of SR. The USM process was optimized using three non-conventional optimization techniques, e.g. ABC, HS and PSO, and it was observed that the performance of those three algorithms was better than that of GA. Rao and Kalyankar (2012) applied TLBO algorithm for parametric optimization of an USM process. Amplitude of vibration, frequency of vibration, mean diameter of abrasive grains, volumetric concentration of abrasive particles in slurry and static feed force were the machining parameters, while MRR was the process performance measure. It was observed that TBLO algorithm had been superior to the other commonly applied non-conventional optimization techniques with respect to optimization performance.

\subsection{Abrasive jet machining process}

In abrasive jet machining (AJM) process, a high velocity jet of abrasive particles and carrier gas coming out from a nozzle strikes on the workpiece surface and erodes it. Material is removed from the workpiece due to erosive action caused by impingement of high velocity jet of abrasive particles. This process is quite suitable for hard and/or brittle metals/alloys, semiconductors and ceramics. For ductile materials, material is removed due to plastic deformation and cutting wear. For brittle materials, material removal mainly takes place due to indentation rupture and elastic-plastic deformation. The important parameters affecting the machining performance of AJM process are the type of the abrasive, its size and concentration, type of the carrier gas, nozzle shape, jet velocity, nozzle pressure and stand-off distance. 
Jain et al. (2007) optimized various machining parameters, like mass flow rate of abrasive particles, mean radius of abrasive particles and velocity of abrasive particles of an AJM process using GA. MRR was maximized subject to SR constraint. Using TLBO algorithm, Rao and Kalyankar (2012) determined the optimal values of mass flow rate of abrasive particles, mean radius of abrasive particles and velocity of abrasive particles of an AJM process while maximizing MRR with respect to SR constraint. It was also observed that the optimization performance of TLBO algorithm had been better than that of GA, SA, ABC, PSO, HS and SFL algorithms.

\subsection{Water jet machining}

The kinetic energy of high speed water jet is used in water jet machining (WJM) process to remove material from the workpiece. The material is eroded from the workpiece surface due to the impact pressure of the water. The potential energy of the water is converted into kinetic energy, with water traveling at a very high speed of about $1000 \mathrm{~m} / \mathrm{sec}$. In pure WJM process, commercially pure water is used (tap water) for machining purpose. The WJM process can be used to machine steel, non-ferrous alloys, polymers, tungsten alloys, concrete, metal matrix composite, glass fibers etc. It can be efficiently applied for drilling, descaling, deburring, cutting of printed circuit boards and profile cutting of fiber-reinforced plastic aircraft structures. This process does not produce any hazardous waste. As water is used as the medium of machining and generally water itself is used for waste removal in other machining processes, so the cost of waste removal is reduced. Its important process parameters include stand-off distance, water pressure, travel speed of jet and nozzle diameter.

Jain et al. (2007) applied GA to search out the optimal parametric combination of a WJM process. Water jet pressure at the nozzle exit, diameter of water jet nozzle, traverse rate of the nozzle and stand-off distance were selected as the important process parameters. MRR was maximized and specific energy was minimized subject to power consumption constraint.

\subsection{Abrasive water jet machining process}

In abrasive water jet machining (AWJM) process, which is a combination of AJM and WJM processes, a stream of small abrasive particles is introduced and entrained in the water jet in such a way that the water jet's momentum is partly transferred to the abrasive particles and those abrasive particles are responsible for removing materials from the workpiece surface. The role of water is primarily to accelerate large quantities of abrasive particles to a high velocity, while generating a highly coherent jet. This process can machine copper and its alloys, aluminum, lead, steel, tungsten carbide, titanium, ceramics, composites, acrylic, concrete, rocks, graphite, silica glass etc. It is often used to machine sandwiched honeycomb structural materials for aerospace industries. Water pressure, water flow rate, type, size and shape of the abrasive particles, flow rate of abrasive particles etc. are observed to be the most important parameters affecting the performance of AWJM process.

Chakravarthy and Rarnesh Babu (1999) used fuzzy logic and GA for parametric optimization of an AWJM process. The best combination of water jet pressure, jet traverse rate and abrasive flow rate values was determined for attaining the desired depth of cut. It was observed that the optimal values of those process parameters could also minimize the cost of production by increasing production rate and reducing the consumption of abrasives, while attaining the desired depth of cut. Chakravarthy and Ramesh Babu (2000) combined fuzzy logic and GA for selecting the optimal parameters, such as water jet pressure, jet traverse rate and abrasive flow rate of an AWJM operation on any material with predetermined thickness. The proposed model would predict the depth of cut achievable with any given set of process parameters. Jain et al. (2007) applied GA to determine the optimal values of water jet pressure at the nozzle exit, diameter of abrasive water jet nozzle, traverse rate of the nozzle, 
mass flow rate of water and mass flow rate of abrasives of an AWJM process to maximize MRR subjected to power consumption constraint.

Srinivasu and Ramesh Babu (2008) developed a neuro-genetic approach to suggest the optimal parameter values (water pressure, abrasive flow rate, jet traverse rate and diameter of focusing nozzle) of an AWJM process for maintaining a desired depth of cut. Zain et al. $\left(2011^{\mathrm{a}}\right)$ integrated ANN and SA techniques to estimate the optimal values of traverse speed, water jet pressure, stand-off distance, abrasive grit size and abrasive flow rate of an AWJM process. SR was selected as the process performance measure. Zain et al. $\left(2011^{\mathrm{b}}\right)$ applied SA and GA techniques for optimization of an AWJM process. The optimal settings of traverse speed, water jet pressure, stand-off distance, abrasive grit size and abrasive flow rate were determined that would lead to the minimum SR value. Zain et al. $\left(2011^{\mathrm{c}}\right)$ considered traverse speed, water jet pressure, stand-off distance, abrasive grit size and abrasive flow rate as the major machining parameters of an AWJM process, and then applied GA and SA techniques to minimize SR of the machined components.

\subsection{Electro jet drilling process}

Electro jet drilling (EJD) is a NTM process where a fine negatively charged stream of acid electrolyte is impinged on the workpiece surface to generate micro-holes. The acid electrolyte is passed under pressure through a finely drawn glass tube nozzle. The electrolyte jet gets charged when a platinum wire inserted into the glass tube is connected to the negative terminal of DC power supply. The workpiece acts as anode. When a suitable electric potential is applied across the two electrodes, the material removal takes place through electrolytic dissolution as the charged electrolyte stream strikes the workpiece. The metal ions thus removed from the workpiece surface are carried away with the flow of the electrolyte. It has several applications, like aviation, space, automobile, electronics and computer, medical, optics and micro-machining.

Sen and Shan (2006) integrated NN, desirability function and GA to search out the optimal values of applied voltage, capillary outside diameter, feed rate, electrolyte concentration and inlet electrolyte pressure of an EJD process. A BPNN was first used to formulate the fitness function for predicting the response parameters of an EJD process. From the network output, the desirability function approach obtained a composite fitness function for further use in GA. The GA then predicted the optimal parametric combinations and optimized the multi-response characteristics of the EJD process. MRR and RE were considered as the responses. Sen and Shan (2007) developed a hybrid NN and GA approach for multi-response optimization of an EJD process. Applied voltage, nozzle diameter, feed rate, electrolyte concentration and inlet electrolyte pressure were the process parameters, and machining rate and SR were the responses.

\subsection{Abrasive flow machining process}

In abrasive flow machining (AFM) process, material is removed by a viscous, abrasive laden semisolid self-deformable grinding media flowing under pressure. The AFM medium is prepared by mixing fine abrasive particles (alumina, silicon carbide, boron carbide, diamond) with a gel-like lubricant to give the desired viscosity. The medium is extruded through or over the workpiece with motion usually in both directions. Places that have the greatest restriction will produce the largest grinding forces. The AFM process acts in a manner similar to grinding or lapping where the extruded abrasive media gently hones edges and surfaces. It is particularly useful when applied to workpieces containing passageways that are considered to be inaccessible to the conventional deburring and polishing tools. This process provides better accuracy, efficiency, economy and possibility of effective automation. Its application areas include aircraft valves bodies and spools, turbine components, automotive parts, finishing of dies, medical instruments, electronic components etc. 
Ali-Tavoli et al. (2007) adopted group method of data handling (GMDH)-type NN and GA to study the effects of number of cycles and abrasive concentration of an AFM process on MRR and SR. Applying real-coded GA, Jain et al. (2007) performed optimization of an AFM process. Concentration of abrasives by volume, abrasive mesh size, number of strokes and extrusion pressure were selected as the predominant AFM process parameters and the objective function was set as to minimize the SR value.

\subsection{Magnetic abrasive finishing process}

In magnetic abrasive finishing (MAF) process, the workpiece to be finished is placeed between two magnetic poles, and the gap between magnetic poles and workpiece is filled with magnetic abrasive particles containing abrasives and ferromagnetic particles. Required finishing pressure is applied by the magnetic field produced by the electromagnets. As a result of this, magnetic abrasives join each other magnetically to form a flexible magnetic abrasive brush for removing peaks of workpiece surface unevenness and thus the workpiece surface is finished. Important machining parameters of MAF process include type and size of magnetic abrasive, concentration of abrasives in ferromagnetic material, gap between workpiece and magnetic poles, magnetic flux density, relative motion between magnetic abrasive particles and workpiece surface, amplitude and frequency of vibrations, and axial movement.

Jain et al. (2007) optimized the values of mean diameter of the magnetic abrasive particles, relative velocity between magnetic abrasive particles and workpiece, volume ratio of ferromagnetic material in the magnetic abrasive powder, input current and finishing time of a MAF process using real-coded GA. Maximization of the difference between initial and final SR values was set as the objective function.

\section{Discussions}

Based on the extensive review on the applications of various non-conventional optimization techniques for parametric optimization of different NTM processes, it is observed that EDM process has been mostly chosen (29\%) by the past researchers for deployment of those optimization tools, as shown in Figure 1. WEDM has been the second preferred $(26 \%)$ NTM process for optimization. The reason for choosing EDM and WEDM processes for parametric optimization may be due to their wide capabilities to machine different complex geometrical features on a wide variety of workpiece materials irrespective of their mechanical properties. These two NTM processes have widespread use in automotive, aerospace, nuclear, mold and die, surgical component, and other manufacturing industries. Although ECDM, LBM and WJM processes have immense potential for industrial applications, little work has been done for optimization of those processes. Figure 2 exhibits the applications of various non-conventional optimization techniques as used for selection of the optimal process parameters of the considered NTM processes.

It is interesting to observe that the applications of GA and NSGA techniques have been mostly preferred by the past researchers. These two techniques together cover almost $68 \%$ of the total applications. It may be due to the availability of GA toolbox in MATLAB software which is easy to comprehend and implement. In other cases (except for SA), the pseudo-codes of the non-conventional optimization algorithms need to be converted into the related MATLAB codes by the process engineers for obtaining the optimal solutions, which probably constrain their applications. In some cases, it is found that GA, NSGA and SA algorithms have been integrated with ANN for process optimization. It is observed that ABC, BBO and TLBO algorithms have also been powerful for NTM process optimization. Mukherjee et al. (2012) compared the optimization performance of GA, PSO, SFA, ACO, ABC and $\mathrm{BBO}$ algorithms, and observed that $\mathrm{BBO}$ algorithm had outperformed the 
others for a WEDM process. As ACO is believed to be a promising optimization algorithm, its performance for NTM process optimization needs to be explored. The importance of various NTM process performance measures as considered by the past researchers for optimization is shown in Figure 3. Majority of the researchers has given maximum importance to MRR (39\%), followed by SR $(34 \%)$. For any machining operation, it is quite obvious that the process engineers should put more emphasis on the amount of material removed from the workpiece per unit of machining time, as the efficiency of any NTM process is directly proportional to MRR. For obtaining accurate shape features on the work materials, SR is the most important criterion. Hence, these two responses of NTM processes have been preferred by the past researchers for subsequent optimization.

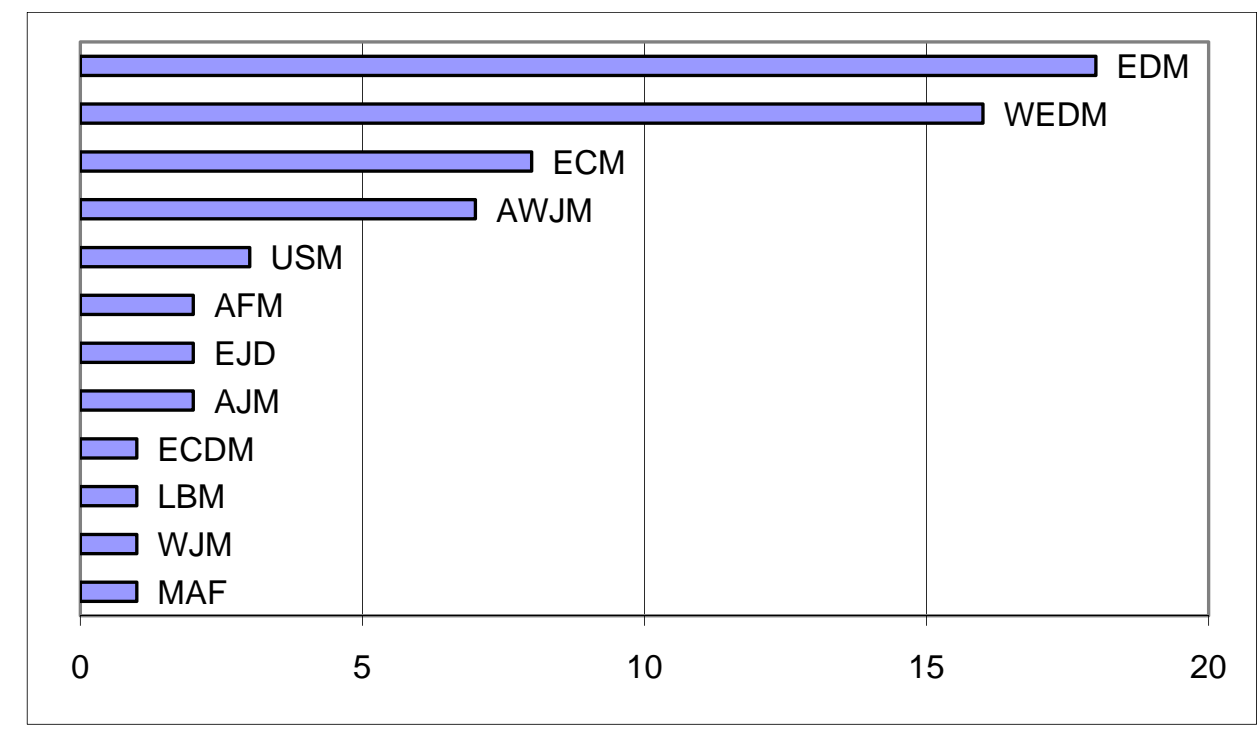

Fig. 1. Optimization of different NTM processes

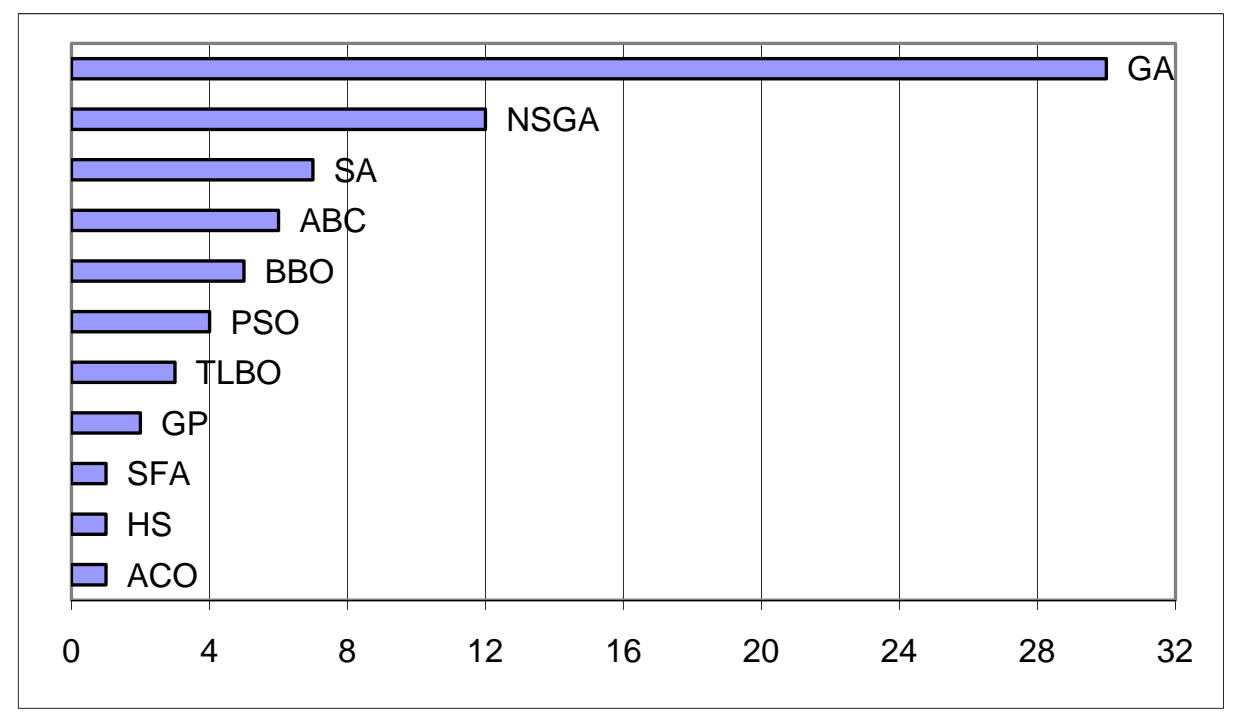

Fig. 2. Applications of various non-conventional optimization techniques

\section{Conclusions}

This paper reviews the applications of various non-conventional optimization techniques for finding out the optimal parametric combinations of different NTM processes. The past researchers have attempted to optimize different NTM processes and it is observed that GA has been the most preferred optimization tool, followed by NSGA. Among the considered NTM processes, EDM process has been selected most number of times for optimization. WEDM has been the second- 
preferred NTM process. MRR and SR have been given maximum importance by the past researchers for optimization as these two responses measure the efficiency and capability of any NTM process. It can be concluded that the non-conventional optimization techniques can be successfully applied for NTM process optimization. These optimization techniques can also be applied to ECDM, LBM, WJM and MAF processes as very little work has been done on their optimization. The applications of ACO and PSO algorithms also need to be explored. As a future scope, the optimization performance of other non-conventional optimization techniques, like gravitational search algorithm, cuckoo search, fire flyer, bat algorithm, fireworks algorithm, differential evolution, society and civilization algorithm etc. may be searched out.

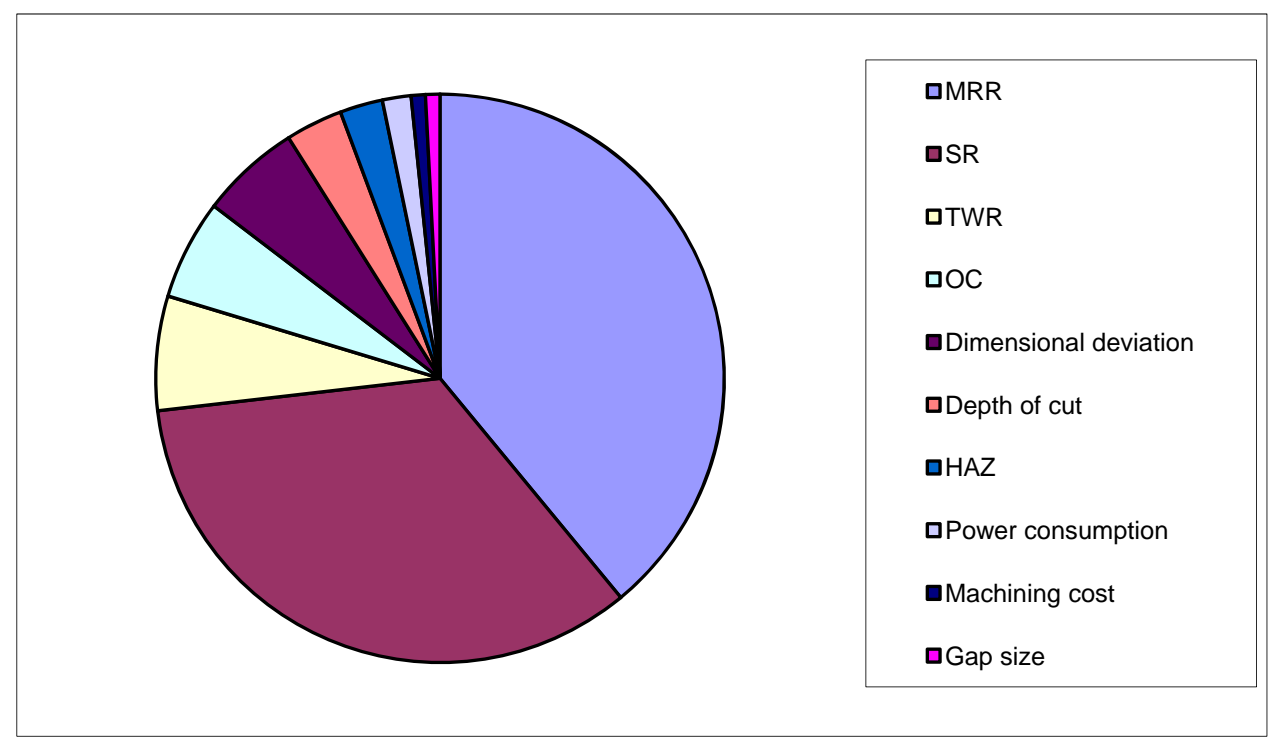

Fig. 3. Importance of NTM process performance measures

\section{References}

Ali-Tavoli, M., Nariman-Zadeh, N., Khakhali, A. \& Mehran, M. (2007). Multi-objective optimization of abrasive flow machining processes using polynomial neural networks and genetic algorithms. Machining Science and Technology, 10, 491-510.

Amini, H., Soleymani Yazdi, M.R. \& Dehghan. (2011). Optimization of process parameters in wire electrical discharge machining of $\mathrm{TiB}_{2}$ nano-composite ceramic. Journal of Engineering Manufacturing, 225(12), 2220-2227.

Bharti, P.S., Maheshwari, S. \& Sharma, C. (2012). Multi-objective optimization of electric-discharge machining process using controlled elitist NSGA-II. Journal of Mechanical Science and Technology, 26(6), 1875-1883.

Chakravarthy, P.S. \& Rarnesh Babu, N. (1999). A new approach for selection of optimal process parameters in abrasive water jet cutting. Materials and Manufacturing Processes, 14(4), 581-600.

Chakravarthy, P.S. \& Rarnesh Babu, N. (2000). A hybrid approach for selection of optimal process parameters in abrasive water jet cutting. Proceedings of the Institution of Mechanical Engineers, Part B: Journal of Engineering Manufacture, 214(9), 781-791.

Chandrasekaran, M., Muralidhar, M., Murali Krishna, C. \& Dixit, U.S. (2010). Application of soft computing techniques in machining performance prediction and optimization: A literature review. International Journal of Advanced Manufacturing Technology, 46, 445-464.

Chen, H-C., Lin, J-C., Yang, Y-K. \& Tsai, C-H. (2010). Optimization of wire electrical discharge machining for pure tungsten using a neural network integrated simulated annealing approach. Expert Systems with Applications, 37, 7147-7153. 
Ciurana, J., Arias, G. \& Ozel, T. (2009). Neural network modeling and particle swarm optimization (PSO) of process parameters in pulsed laser micromachining of hardened AISI H13 steel. Materials and Manufacturing Processes, 24(3), 358-368.

Datta, D. \& Das, A.K. (2010). Tuning process parameters of electrochemical machining using a multi-objective genetic algorithm: A preliminary study. In Lecture notes in Computer Science, Deb, K. et al. (Eds.) Springer-Verlag Berlin Heidelberg, 485-493.

Fenggou, C. \& Dayong, Y. (2004). The study of high efficiency and intelligent optimization system in EDM sinking process. Journal of Materials Processing Technology, 149, 83-87.

Gao, Q., Zhang, Q-H., Su, S-P. \& Zhang, J-H. (2008). Parameter optimization model in electrical discharge machining process. Journal of Zhejiang University Science A, 9(1), 104-108.

Golshan, A., Gohari, S. \& Ayob, A. $\left(2011^{\mathrm{a}}\right)$. Computational intelligence in optimization of wire electrical discharge machining of cold-work steel 2601. International Journal of Mechanical \& Mechatronics Engineering, 11(4) 14-19.

Golshan, A., Gohari, S. \& Ayob, A. $\left(2011^{\mathrm{b}}\right)$. Comparison of intelligent optimization algorithms for wire electrical discharge machining parameters. Proc. of $3^{\text {rd }}$ International Conference on Computational Intelligence, Modelling \& Simulation, Malaysia, 134-140.

Jain, N.K., Jain, V.K. \& Deb, K. (2007). Optimization of process parameters of mechanical type advanced machining processes using genetic algorithms. International Journal of Machine Tools \& Manufacture, 47(6), 900-919.

Jain, N.K., Jain, V.K. \& Jha, S. (2007). Parametric optimization of advanced fine-finishing processes. International Journal of Advanced Manufacturing Technology, 34, 1191-1213.

Jain, N.K. \& Jain, V.K. (2012). Optimization of electro-chemical machining process parameters using genetic algorithms. Machining Science and Technology, 11(2), 235-258.

Joshi, S.N. \& Pande, S.S. (2011). Intelligent process modeling and optimization of die-sinking electric discharge machining. Applied Soft Computing, 11, 2743-2755.

Kanagarajan, D., Karthikeyan, R., Palanikumar, K. \& Davim, J.P. (2008). Optimization of electrical discharge machining characteristics of $\mathrm{WC} / \mathrm{Co}$ composites using non-dominated sorting genetic algorithm (NSGA-II). International Journal of Advanced Manufacturing Technology, 36, 11241132.

Kolahan, F. \& Bironro, M. (2008). Modeling and optimization of process parameters in PMEDM by genetic algorithm. World Academy of Science, Engineering and Technology, 48, 480-484.

Kondayya, D. \& Gopala Krishna, A. (2011). An integrated evolutionary approach for modelling and optimization of wire electrical discharge machining. Proceedings of the Institution of Mechanical Engineers, Part B: Journal of Engineering Manufacture, 225(4), 549-567.

Kumar, K. \& Agarwal, S. (2011). Multi-objective parametric optimization on machining with wire electric discharge machining. International Journal of Advanced Manufacturing Technology, DOI 10.1007/s00170-011-3833-1

Kuriakose, S. \& Shunmugam, M.S. (2005). Multi-objective optimization of wire-electro discharge machining process by non-dominated sorting genetic algorithm. Journal of Materials Processing Technology, 170, 133-141.

Kuruvila, N. \& Ravindra, H.V. (2011). Parametric influence and optimization of wire EDM of hot die steel. Machining Science and Technology, 15(1), 47-75.

Mahapatra, S.S. \& Patnaik, A. (2006). Optimization of wire electrical discharge machining (WEDM) process parameters using genetic algorithm. Indian Journal of Engineering \& Materials Sciences, 13, 494-502.

Maji, K. \& Pratihar, D.K. (2011). Modeling of electrical discharge machining process using conventional regression analysis and genetic algorithms. Journal of Materials Engineering and Performance, 20, 1121-1127.

Majumder, A. (2012). Parametric optimization of electric discharge machining by GA-based response surface methodology. Journal for Manufacturing Science and Production, 12, 25-30. 
Mandal, D., Pal, S.K. \& Saha, P. (2007). Modeling of electrical discharge machining process using back propagation neural network and multi-objective optimization using non-dominating sorting genetic algorithm-II. Journal of Materials Processing Technology, 186, 154-162.

Mukherjee, R. \& Chakraborty, S. (2012). Selection of the optimal electrochemical machining process parameters using biogeography-based optimization algorithm. International Journal of Advanced Manufacturing Technology, DOI 10.1007/s00170-012-4060-0.

Mukherjee, R. \& Chakraborty, S. (2012). Selection of EDM process parameters using biogeographybased optimization algorithm. Materials and Manufacturing Processes, 27, 954-962.

Mukherjee, R., Chakraborty, S. \& Samanta, S. (2012). Selection of wire electrical discharge machining process parameters using non-traditional optimization algorithms. Applied Soft Computing, 12, 2506-2516.

Nejad, R.A.M. (2011). Modeling and optimization of electrical discharge machining of SiC parameters, using neural network and non-dominating sorting genetic algorithm (NSGA II), Materials Sciences and Applications, 2, 669-675.

Padhee, S., Nayak, N., Panda, S.K., Dhal, P.R. \& Mahapatra, S.S. (2012). Multi-objective parametric optimization of powder mixed electro-discharge machining using response surface methodology and non-dominated sorting genetic algorithm. Sadhana, 37(2), 223-240.

Rao, G.K.M., Rangajanardhaa, G., Rao, D.H. \& Rao, M.S. (2009). Development of hybrid model and optimization of surface roughness in electric discharge machining using artificial neural networks and genetic algorithm. Journal of Materials Processing Technology, 209, 1512-1520.

Rao, R.V., Pawar, P.J. \& Shankar, R. (2008). Multi-objective optimization of electrochemical machining process parameters using a particle swarm optimization algorithm. Proceedings of the Institution of Mechanical Engineers, Part B: Journal of Engineering Manufacture, 222(8), 949958.

Rao, R.V. \& Pawar, P.J. (2009). Modelling and optimization of process parameters of wire electrical discharge machining. Proceedings of the Institution of Mechanical Engineers, Part B: Journal of Engineering Manufacture, 223(11), 1431-1440.

Rao, R.V., Pawar, P.J. \& Davim, J.P. (2010). Parameter optimization of ultrasonic machining process using nontraditional optimization algorithms. Materials and Manufacturing Processes, 25(10), $1120-1130$.

Rao, R.V. \& Kalyankar, V.D. (2012). Parameter optimization of modern machining processes using teaching-learning-based optimization algorithm. Engineering Applications of Artificial Intelligence, doi: 10.1016/j.engappai.2012.06.007.

Salman, Ö. \& Kayacan, M.C. (2008). Evolutionary programming method for modeling the EDM parameters for roughness. Journal of Materials Processing Technology, 200, 347-355.

Samanta, S. \& Chakraborty, S. (2011). Parametric optimization of some non-traditional machining processes using artificial bee colony algorithm. Engineering Applications of Artificial Intelligence, 24(6), 946-957.

Sen, M. \& Shan, H.S. (2006). Optimal selection of machining conditions in the electrojet drilling process using hybrid NN-DF-GA approach. Materials and Manufacturing Processes, 21, 349-356.

Sen, M. \& Shan, H.S. (2007). Electro jet drilling using hybrid NNGA approach. Robotics and Computer-Integrated Manufacturing, 23, 17-24.

Senthilkumar, C., Ganesan, G. \& Karthikeyan, R. (2010). Bi-performance optimization of electrochemical machining characteristics of $\mathrm{Al} / 20 \% \mathrm{SiCp}$ composites using NSGA-II. Proceedings of the Institution of Mechanical Engineers, Part B: Journal of Engineering Manufacture, 224(9), 1399-1407.

Senthilkumar, C., Ganesan, G. \& Karthikeyan, R. (2011). Parametric optimization of electrochemical machining of $\mathrm{Al} / 15 \% \mathrm{SiCp}$ composites using NSGA-II. Transactions of Nonferrous Metals Society of China, 21, 2294-2300. 
Somashekhar, K.P., Ramachandran, N. \& Mathew, J. (2010). Optimization of material removal rate in micro-EDM using artificial neural network and genetic algorithms. Materials and Manufacturing Processes, 25, 467-475.

Somashekhar, K.P., Mathew, J. \& Ramachandran, N. (2012). A feasibility approach by simulated annealing on optimization of micro-wire electric discharge machining parameters. International Journal of Advanced Manufacturing Technology, DOI 10.1007/s00170-012-4096-1.

Srinivasu, D.S. \& Ramesh Babu, N. (2008). A neuro-genetic approach for selection of process parameters in abrasive waterjet cutting considering variation in diameter of focusing nozzle. Applied Soft Computing, 8, 809-819.

Su, J.C., Kao, J.Y. \& Tarng, Y.S. (2004). Optimisation of the electrical discharge machining process using a GA-based neural network. International Journal of Advanced Manufacturing Technology, 24, 81-90.

Tarng. Y.S., Ma, S.C. \& Chung, L.K. (1995). Determination of optimal cutting parameters in wire electrical discharge machining. International Journal of Machine Tools \& Manufacture, 35(12), 1693-1701.

Yang, S-H., Srinivas, J., Mohan, S., Lee, D-M. \& Balaji, S. (2009). Optimization of electric discharge machining using simulated annealing. Journal of Materials Processing Technology, 209, 44714475.

Yang, R.T., Tzeng, C.J., Yang, Y.K. \& Hsieh, M.H. (2012). Optimization of wire electrical discharge machining process parameters for cutting tungsten. International Journal of Advanced Manufacturing Technology, 60, 135-147.

Yusup, N., Zain, A.M., Zaiton, S. \& Hashim, M. (2012). Evolutionary techniques in optimizing machining parameters: Review and recent applications (2007-2011). Expert Systems with Applications, 39, 9909-9927.

Zain, A.M., Harona, H. \& Sharif, S. $\left(2011^{\mathrm{a}}\right)$. Estimation of the minimum machining performance in the abrasive waterjet machining using integrated ANN-SA. Expert Systems with Applications, 38, 8316-8326.

Zain, A.M., Haron, H. \& Sharif, S. $\left(2011^{\mathrm{b}}\right)$. Optimization of process parameters in the abrasive waterjet machining using integrated SA-GA. Applied Soft Computing, 11, 5350-5359.

Zain, A.M., Haron, H. \& Sharif, S. $\left(2011^{c}\right)$. Genetic algorithm and simulated annealing to estimate optimal process parameters of the abrasive waterjet machining. Engineering with Computers, 27, 251-259. 